\title{
A promising alternative anti-HBV agent: The targeted ribonuclease
}

\author{
YINGHUI LI, YA ZHAO, JUN LIU, YUXIAO HUANG, ZHONGXIANG LIU and CAIFANG XUE \\ Department of Medical Microbiology and Parasitology, Fourth Military Medical University, \\ Xi'an, Shaanxi 710032, P.R. China
}

Received January 25, 2010; Accepted March 18, 2010

DOI: 10.3892/ijmm_00000434

\begin{abstract}
HBV-targeted ribonuclease (TR) is a fusion of HBV core protein $(\mathrm{HBVc})$ and human eosinophil-derived neurotoxin (hEDN). Introduction of TR by transfection or transduction into HepG2.2.15 cells (a cell model of HBV infection) revealed that it significantly reduces serological markers of $\mathrm{HBV}$ replication (including $\mathrm{HBsAg}, \mathrm{HBeAg}$ and HBV DNA) in cell supernatants, suggesting that the targeted ribonuclease inhibits $\mathrm{HBV}$ replication. To further our understanding of the molecular mechanism of the anti-HBV effect of TR, we expressed TR in E. coli and found that purified TR possesses RNase activity and targeting activity. Furthermore, the antiviral effect of TR depends both on an enzymatically active hEDN and on the core domain. In or out of HepG2.2.15 cells, TR coassembles with the wild-type capsid protein into particles with internal hEDN domains. Our data suggest an intracellular ribonuclease activation mechanism that, owing to the characteristics of HBV morphogenesis, is highly virus specific. HBV may therefore be particularly vulnerable to the capsid-targeted viral inactivation approach.
\end{abstract}

\section{Introduction}

Hepatitis B virus (HBV) is a small DNA virus that replicates via reverse transcription and causes acute and chronic B-type hepatitis in humans. Chronic HBV infection is a major health problem worldwide. Globally, more than 350 million people are infected with $\mathrm{HBV}$, and some of them will develop liver cirrhosis and hepatocellular carcinoma (HCC). Current treatment regimens for chronic $\mathrm{HBV}$ infection, including interferon- $\gamma$, lamivudine, adefovir and different combinations of these drugs, have only a limited long-term efficacy and are associated with many adverse effects and drug resistance

Correspondence to: Dr Caifang Xue, Department of Medical Microbiology and Parasitology, Fourth Military Medical University, Xi'an, Shaanxi 710032, P.R. China

E-mail: caifangxue@fmmu.edu.cn

Key words: targeted ribonuclease, hepatitis B virus, therapy
$(1,2)$. Therefore, the discovery of novel treatment strategies for HBV infection is both necessary and urgent. In fact, many novel treatment strategies have been tested for inhibition of HBV replication, such as antisense nucleotides, ribozymes, intracellular antibodies, targeted nucleases and RNA interference (3-13). All of these inhibit HBV replication to various degrees.

To explore alternative treatments for HBV infection, we previously constructed HBV-targeted ribonuclease (TR), a fusion protein of $\mathrm{HBV}$ core protein $(\mathrm{HBVc})$ and human eosinophil-derived neurotoxin (hEDN), and its effect on HBV replication was tested (14). After the targeted ribonuclease was introduced by transfection or transduction into HepG2.2.15 cells (a cell model of HBV infection), we found that it significantly reduced serological markers of $\mathrm{HBV}$ replication, namely $\mathrm{HBsAg}, \mathrm{HBeAg}$, and $\mathrm{HBV}$ DNA in the supernatants of HepG2.2.15 cells, suggesting that the targeted ribonuclease inhibited $\mathrm{HBV}$ replication.

In the present study, we explored the molecular mechanisms of the anti-HBV effect of the targeted ribonuclease and its dependencies. We found that purified TR possesses RNase and targeting activities in vitro, which supports the following mechanism: TR specifically recognizes pregenomic RNA (pgRNA) of HBV (the template for HBV replication) intracellularly via the $\mathrm{HBVc}$ domain, and the hEDN domain degrades the pgRNA. This leads to reduced replication of HBV DNA and reduced synthesis of viral proteins, which finally results in a reduction in extracellular HBV DNA and viral proteins HBsAg and HBeAg. Together with our previous findings, this clarifies the mechanism by which HBV replication is inhibited by the TR. The targeted ribonuclease may therefore be a promising alternative antiHBV agent.

We report that the antiviral strategy of capsid-targeted viral inactivation (CTVI) was designed to disable newly produced virions by fusing a degradative enzyme (e.g., a nuclease or protease) to a viral protein that causes the degradative enzyme to be inserted into virions during assembly. Several new experimental approaches have been developed that increase the antiviral effect of the CTVI strategy on retroviral and retroviral-like replication in vitro, such as that of A Moloney murine leukemia virus, dengue 2 virus and HBV. We recently demonstrated the principle of CTVI against HBV infection and observed a modest therapeutic effect in vivo. 


\section{Materials and methods}

Construction of plasmids. All the recombinant DNA and plasmid constructs were prepared using standard subcloning procedures by inserting fragments. The coding genes of hEDN, TR and TRmut in pcDNA3.1(-)/hEDN, pcDNA3.1(-)/ TR and pcDNA3.1(-)/TRmut [hEDN mutated for just one amino acid, Lys113 $\rightarrow$ Arg, which eliminates the ribonuclease activity (35)] were cloned into prokaryotic expression vector pET32a(+). After digesting with BamHI and HindIII, we verified the successful construction of pET32a(+)/hEDN, pET32a(+)/TR and pET32a(+)/TRmut.

Protein expression, purification and determination. BL21(DE3) E. coli cells were freshly transformed with the pET32a(+)/hEDN, pET32a(+)/TR and pET32a(+)/TRmut plasmid and fermented as recommended by the supplier. The three types of fusion protein were isolated from inclusion bodies, denatured, renatured and dialyzed as described (36). Fractions containing hEDN, TR and TRmut were pooled, dialyzed against $10 \mathrm{mM}$ Tris- $\mathrm{HCl}(\mathrm{pH} 7.5), 25 \mathrm{mM} \mathrm{NaCl}$, and lyophilized. The fractions containing hEDN, TR and TRmut were constructed in $0.8 \mathrm{mM}$ imidazole and $1 \%$ Triton X-100 (Sigma, St. Louis, MO, USA) before $0.6 \mathrm{ml}$ of $\mathrm{Ni}^{2+}$ NTA-agarose was added. The slurry was rotated end over end for $1 \mathrm{~h}$ at room temperature before collection on a column. The column was washed with $20 \mathrm{ml}$ of $20 \mathrm{mM}$ Tris- $\mathrm{HCl}$ (pH 7.5) containing $0.8 \mathrm{mM}$ imidazole and step-eluted with 4-column volumes each of the same buffer with 40, 50, 60, 100, 200, 300 and $400 \mathrm{mM}$ imidazole. Protein was monitored throughout the purification procedure using the BCA protein assay reagent (Pierce, Rockford, IL, USA) according to the manufacturer's instructions using bovine albumin as standard.

Ribonuclease assay. Two types of methods were used to detect the ribonuclease activity of TR. Total RNA was prepared from HepG2 cells by using the Trizol RNA Isolation reagent (Invitrogen, Carlsbad, CA, USA) according to the manufacturer's protocol. Four micrograms of total cellular RNA was incubated with $1 \mu \mathrm{g}$ of TR protein sample in diethyl pyrocarbonate water at $37^{\circ} \mathrm{C}$ for $1 \mathrm{~h}$. RNase activity was measured by the degradation of intact total cellular RNA by formaldehyde agarose gel (1.2\%). The RNA samples were electrophoresed at $50 \mathrm{~V}$ for $90 \mathrm{~min}$, stained with ethidium bromide and visualized by UV illumination. In the other method used to detect ribonuclease activity, TR and hEDN were measured in a final volume of $0.3 \mathrm{ml}$ containing $0.33 \mathrm{mg} /$ $\mathrm{ml}$ yeast tRNA, 0.17 M Tris-HCl (pH 7.5), 0.17 mM EDTA, $0.17 \mathrm{mg} / \mathrm{ml}$ human serum albumin (Calbiochem, Los Angeles, CA, USA) and the appropriate concentration of the ribonuclease (dilutions were constructed in $0.5 \mathrm{mg} / \mathrm{ml}$ human serum albumin). The mixtures were incubated for $15 \mathrm{~min}$ at $37^{\circ} \mathrm{C}$ before termination with $700 \mu 1$ of $3.4 \%$ ice-cold perchloric acid. All assays were performed in the linear range of the enzyme except where noted. Absorbance readings of the appropriate blanks were subtracted from assays containing the enzyme.

Cell culture, transfection, $\beta$-galactosidase $(\beta$-Gal) activity assay and fluorescence microscopy. HepG2.2.15 cells were maintained in DMEM supplemented with $10 \%$ fetal bovine serum. Transfections of the plasmids pcDNA3.1(-)/hEDN, pcDNA3.1(-)/TR and pcDNA3.1(-)/TRmut were performed as previously described (14). Transgene expression was determined by fluorescence microscopy. For the B-Gal reporter gene assay, the total amount of DNA used in each transfection was kept constant by the addition of appropriate amounts of empty vector pcDNA3.1(-), and assays were performed according to the manufacturer's protocol. B-Gal activity was normalized to the protein concentration in each well. All transfection experiments were repeated at least twice with triplicate wells.

Targeting activity of the $H B V$-targeted ribonuclease. The targeting activity of the $\mathrm{HBV}$-targeted ribonuclease was assayed both in and out of the transfection experiment. After transfection, the total RNA of the transfected groups was extracted with Trizol, while HepG2.2.15 cell extracts were prepared and subsequently incubated with $0.2 \mathrm{nM}$ TR, TRmut and hEDN, respectively, at $37^{\circ} \mathrm{C}$ for $1 \mathrm{~h}$. Viral RNA was isolated using the QIAmp Viral RNA Minikit (Qiagen, Valencia, CA, USA). The total RNA of the transfection groups and the viral RNA of the incubation groups were used as the template for RT-PCR, which was carried out to detect the degradation of the HBV pregenomic RNA. The primers to the HBV pregenomic RNA were designed as reported (17).

Statistical analysis. Analysis of variance and the Student's t-test were used for data analysis. Differences were considered significant at $\mathrm{P}<0.05$.

\section{Results}

Expression and purification of hEDN, TR and TRmut. hEDN, TR and TRmut were expressed in E. coli [BL21(DE3)]. Induction with isopropyl-1-thio- $\beta$-D-galactopyranoside (IPTG) led to an accumulation of the expressed protein in the inclusion bodies. In the inclusion bodies, hEDN represented $\sim 50 \%$ of the protein (apparent molecular mass, $25 \mathrm{kDa}$ ) (Fig. 1, lanes 2 and 5). Although TR and TRmut were not expressed in as high a yield as hEDN, it still represented one of the major components of the inclusion bodies (Fig. 1, lanes 1 and 4, lanes 3 and 6, respectively). In SDS-polyacrylamide gel electrophoresis, TR and TRmut migrated with an apparent molecular mass of $43 \mathrm{kDa}$, which was close to the expected size (the calculated molecular mass of TR is 45,048 Daltons). The inclusion bodies were vigorously washed, and the proteins were denatured with $8 \mathrm{M}$ urea and renatured by rapid dilution into Refolding buffer (0.25 mM Tris- $\mathrm{HCl}$ ( $\mathrm{pH} 7.5), 0.1 \mathrm{M}$ $\mathrm{NaCl}, 1.0 \mathrm{M}$ urea and $10 \%$ glycerol). After exhaustive dialysis in Native lysis buffer (Qiagen), the renatured proteins were added to $0.6 \mathrm{ml}$ of $\mathrm{Ni}^{2+}$-NTA-agarose, incubated and washed using the same buffer with 40, 50, 60, 100, 200, 300 and $400 \mathrm{mM}$ imidazole. This resulted in the elimination of the majority of contaminating proteins. Only 5\% of the total protein found in the inclusion bodies was purified as functional protein. Large losses occurred at both the renaturing step and at the dialysis step. Similar observations have been reported $(15,16)$. 


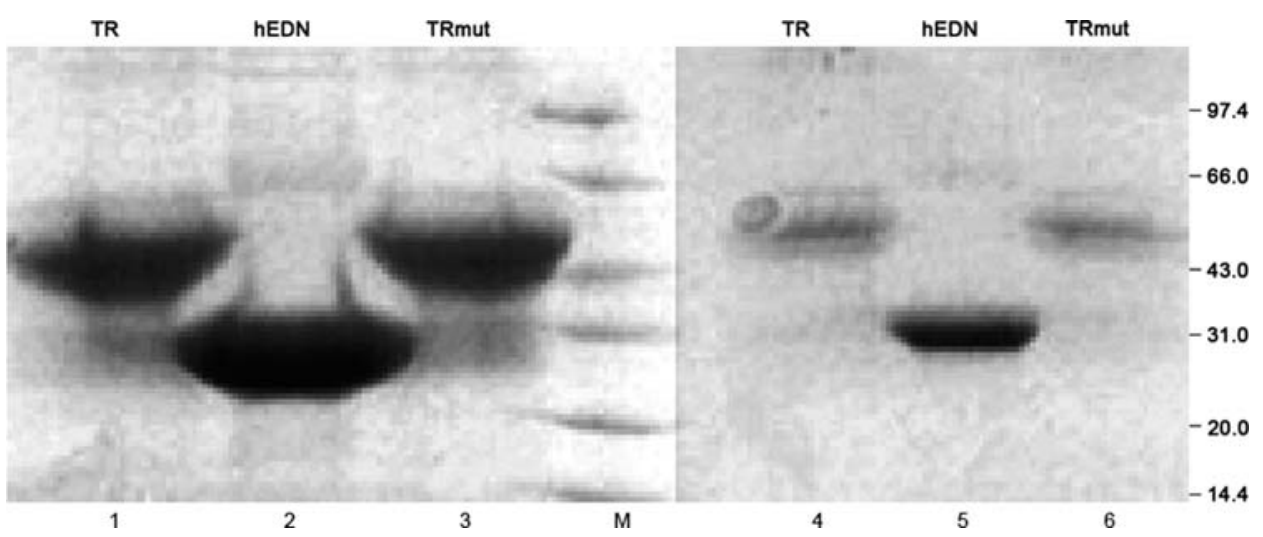

Figure 1. SDS-polyacrylamide gel electrophoresis of TR, hEDN and TRmut. Lanes 1-3 are stained with Coomassie Blue, lanes 4-6 show Western blotting with polyclonal rabbit anti-TR. Lane M shows the molecular mass markers indicating the sizes 97.4, 66.0, 43.0, 31.0, 20.0 and 14.4 kDa from top to bottom.

Ribonuclease activity. Authentic TR exhibits specific RNase activity, including digestion of RNA isolated from HepG2 cells. Fig. 2A shows the RNase activity of TR against RNA isolated from HepG2 cells. Based on the degradation of RNA as analyzed by electrophoresis in $1.2 \%$ formaldehyde agarose gels, TR exhibited potent RNase activity, whereas no RNase activity was detected in TRmut. Fig. 2A also shows the dosedependent RNase activity of TR. Complete digestion of both substrates was obvious at $1.0 \mu \mathrm{g}$ of TR, and cleavages were apparent at $0.4 \mu \mathrm{g}$. The ribonuclease activity of TR and TRmut was measured and compared to that of hEDN. The enzymatic activities of both TR and hEDN were inhibited by placental ribonuclease inhibitor (data not shown) and by an anti-TR antibody similarly. As shown in Fig. 2B, TR exhibited ribonuclease activity that was inhibited by the antiTR antibody. The specific activity of hEDN, however, was 6$13 \%$ that of TR. The lower specific activity of the hEDN protein could be due either to improper folding or to the coupling of the $\mathrm{C}$ terminus of the ribonuclease to another protein interfering with its enzymatic activity. At present, there is no way to distinguish these possibilities or to separate the active from the inactive protein. All subsequent concentrations of TR reported in this study reflect the protein concentration determined by the ribonuclease activity. Thus, TR retains both functionalities of the fusion protein: ribonuclease activity and the ability to package into HBV capsid (data not shown).

Assay of TR targeting activity. As shown in Fig. 3A, the results of the B-Gal activity assays indicate that the transfection efficiency of HepG2.2.15 cells (human hepatocellular liver carcinoma cell line transfected with hepatitis B virus DNA; from ATCC) transfected with pcDNA3.1(-)/TRmut, pcDNA3.1(-)/hEDN, pcDNA3.1(-) or mock transfection did not significantly different from each other $(\mathrm{P}>0.05)$. As shown in Fig. 3B, transgene expression was confirmed in the transfected HepG2.2.15 cell line by fluorescence microscopy. Forty-eight hours after the transfection, all cells were fixed in $20 \mathrm{~g} / 1$ paraformaldehyde and $1 \mathrm{~g} / 1$ Triton X-100 diluted in $\mathrm{PBS}$ and put on ice for $30 \mathrm{~min}$. After incubating with rabbit anti-TR Ab and goat anti-rabbit IgG labeled with FITC, the cells were observed by fluorescence microscopy. Upon fluorescence microscopy, abundant fluorescence was noted in the cytoplasm of cells transfected with pcDNA3.1(-)/TR, pcDNA3.1(-)/TRmut and pcDNA3.1(-)/hEDN, but no fluorescence was found in the control cells. This result clearly suggests that the TR transgene was expressed in the HepG2.2.15 cells with high efficiency. The concentrations of HBsAg and HBeAg in the cell extracts transfected with pcDNA3.1(-)/TR were significantly lower than those in the controls $(\mathrm{P}<0.05)$, while these concentrations in HepG2.2.15 cells transfected with pcDNA3.1(-)/TRmut, pcDNA3.1(-)/ hEDN, pcDNA3.1(-), or mock transfection did not differ significantly from each other $(\mathrm{P}>0.05)$. Compared with those of the mock transfected HepG2.2.15 cells, the concentrations of $\mathrm{HBsAg}$ and $\mathrm{HBeAg}$ in the cells transfected with pcDNA3.1(-)/TR were lower by 31 and $41 \%$, respectively (Fig. 3C).

On the basis of the above results, the targeting activity of TR was assessed further. Utilizing the unique structure of the HBV genome and transcripts, a rapid transcript-specific RT-PCR assay was developed that allowed the specific detection of preC mRNA molecules and simultaneously monitored the level of total RNA transcription (pre-C mRNA plus pgRNA) (17). First, lysates of the HepG2.2.15 cells were prepared and co-incubated with TR. Then RNA in the lysate was extracted as a template, and RT-PCR was carried out to detect the degradation of 3.5-kb RNA. As shown in Fig. 4A, with no difference in the housekeeping gene GAPDH, the loss of 3.5-kb RNA due to co-incubation with TR was significantly greater than that of the controls, and coincubation with TRmut and hEDN resulted in no difference from the negative control. Furthermore, we found increasing degradation of 3.5-kb RNA with increasing TR concentrations (Fig. 4B). Next, we assayed the targeting activity of TR in a transfection experiment. Scanning of electrophoresis showed that transfection with TR significantly decreased the 3.5-kb RNA levels (Fig. 4C). Prior to this report, we demonstrated that the targeted ribonuclease constructed by us (the fusion protein of $\mathrm{HBVc}$ and $\mathrm{hEDN}$ ) dramatically reduced the concentration of $\mathrm{HBsAg}, \mathrm{HBeAg}$ and $\mathrm{HBV}$ DNA in the supernatant of transfected HepG2.2.15 cells $(14,18)$. Although it is possible that the decrease was caused by factors other than the inhibition of $\mathrm{HBV}$ replication by targeted 
A

B
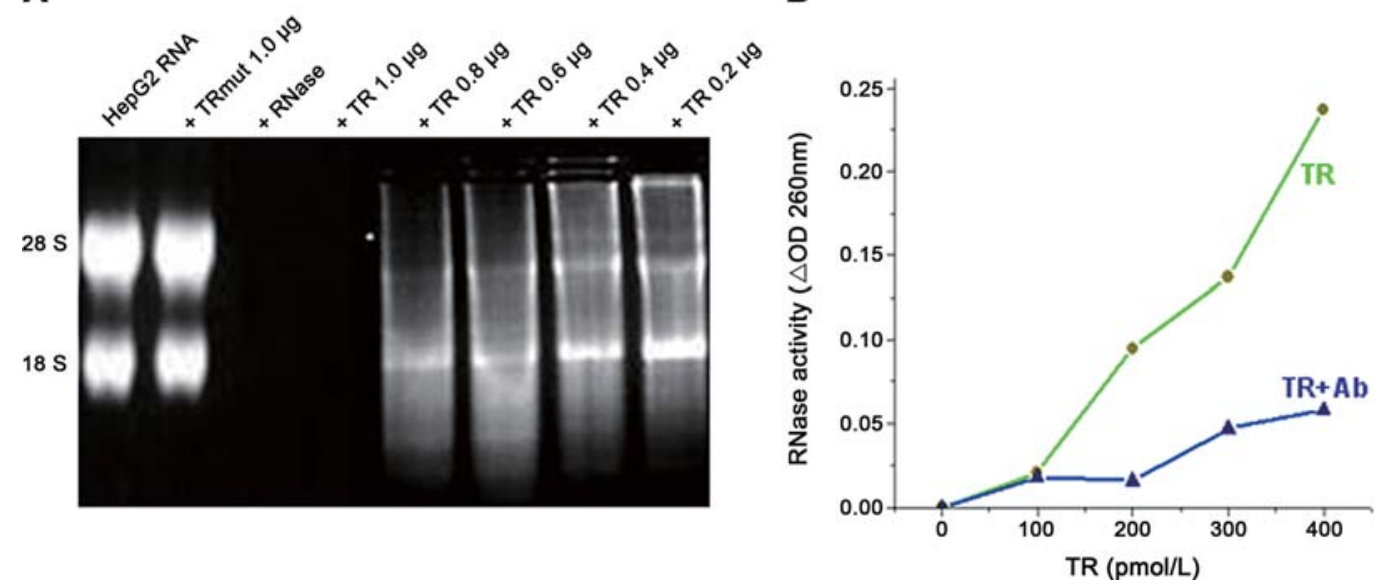

Figure 2. RNase activity of TR. (A) RNase activity of TR was assayed by the degradation of total RNA isolated from HepG2 cells. TR demonstrated a potent dose-dependent RNase activity comparable to that of bovine pancreatic RNase A, whereas TRmut showed no RNase activity. (B) Inhibition of the ribonuclease activity of TR by an anti-TR antibody. Enzyme activity was assayed as described in Materials and methods using yeast tRNA as a substrate. Polyclonal anti-TR antibody was added to the indicated concentrations of TR, and all tubes including those without antibody addition were preincubated for $1 \mathrm{~h}$ at $4^{\circ} \mathrm{C}$ before assaying at $37^{\circ} \mathrm{C}$ for $15 \mathrm{~min}$. This assay was not performed in the linear range of the assay. The linear range of TR enzymatic activity is between $10-100 \mathrm{pM}$ TR.

A

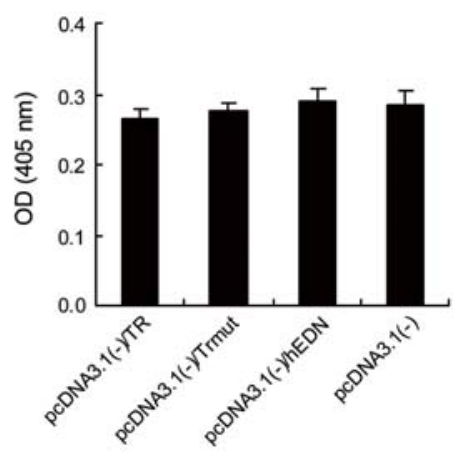

C

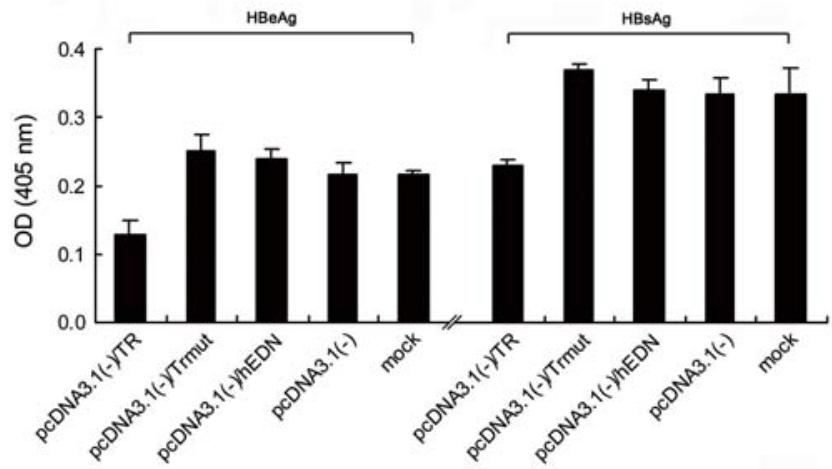

B
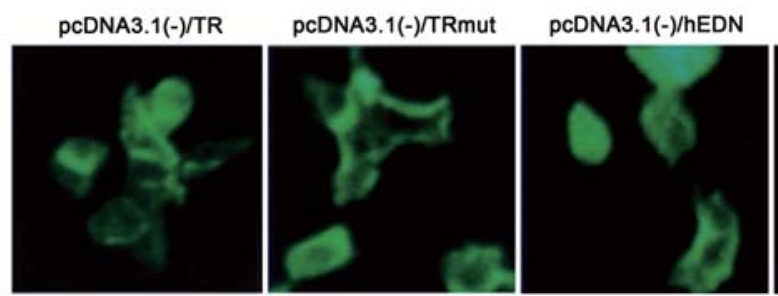

pcDNA3.1(-)

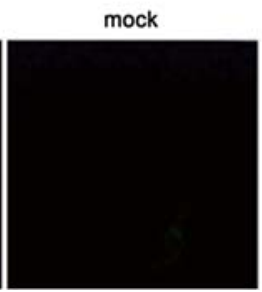

Figure 3. Detection of the transfection and the antiviral effect of TR. (A) Relative B-Gal activity of co-transfections was detected in the lysates of the transfected HepG2.2.15 cells (left) and expressed in OD $405 \mathrm{~nm}$ values as the means \pm SD of three independent assays. (B) Upon fluorescence microscopy, abundant fluorescence was noted in the cytoplasm of the cells transfected with pcDNA3.1(-)/TR, pcDNA3.1(-)/TRmut and pcDNA3.1(-)/hEDN, whereas no fluorescence was noted in the control cells. (C) TR anti-HBV activity was determined by HBsAg and HBeAg in the lysates of HepG2.2.15 cells. As compared to the mock group, the concentrations of HBsAg and HBeAg in the pcDNA3.1(-)/TR transfection group decreased by 31 and $41 \%$, respectively.

ribonuclease, our results strongly disprove this argument. First, the fact that pcDNA3.1(-)/TR inhibited HBV replication while pcDNA3.1(-)/TRmut (which is identical to pcDNA3.1(-)/TR except for one amino acid mutation that eliminates ribonuclease activity) did not indicates that the reduction was dependent on the ribonuclease activity of the targeted ribonuclease. Second, transfection with pcDNA3.1(-)/
hEDN did not affect the results, showing that the reduction relies on $\mathrm{HBVc}$ as a targeting molecule in the fusion protein.

\section{Discussion}

Hepatitis B virus infection (HBV) is a significant global health problem. Despite the success of universal hepatitis B 
A

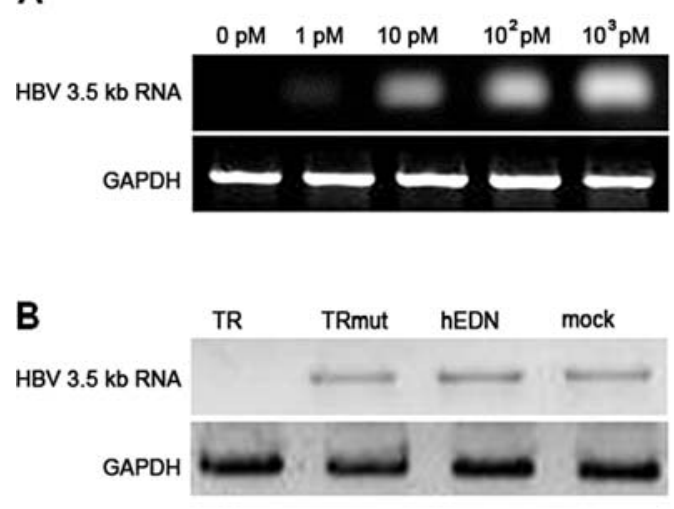

C
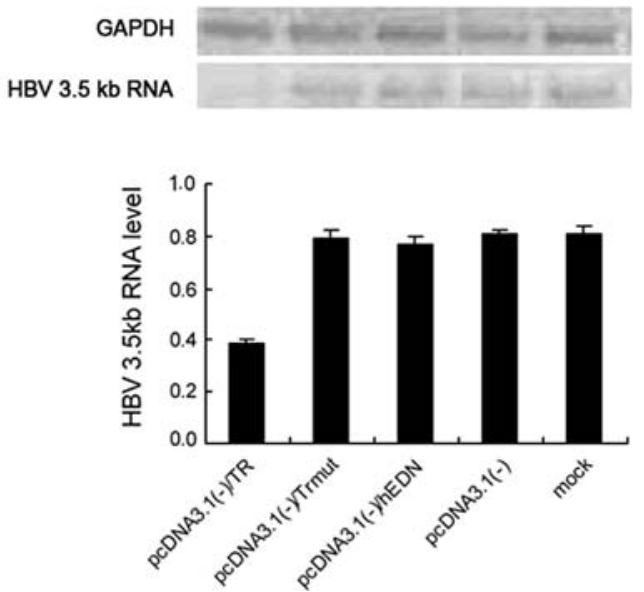

Figure 4. Detection of the targeting activity of TR. (A) HepG2.2.15 cell lysates were prepared and co-incubated with TR. With no difference in the housekeeping gene GAPDH, the loss of the 3.5-kb RNA from co-incubation with TR was significantly higher than in the controls, and that from coincubation with TRmut and hEDN was not different from the negative control. (B) Increasing degradation of the 3.5-kb RNA with increasing TR concentratio was noted. (C) The targeting activity of TR was detected in a transfection experiment. Scanning of electrophoresis showed that transfection with TR significantly decreased the level of 3.5-kb RNA.

vaccination in many countries, more than 350 million individuals worldwide are chronically infected and $15-40 \%$ of those will develop cirrhosis and/or hepatocellular carcinoma if left untreated. Available therapies for chronic HBV infection are effective at decreasing viremia and improving measured clinical outcomes. However, no single therapy is optimal. As such, alternative drug therapies and investigations of their role in the management of chronic HBV are warranted. Significant improvements in the understanding of the HBV life cycle, viral genomics, and virushost interactions continue to lead to the development of novel viral targets and immune modulators. The restriction of HBV genome replication to the nucleocapsid makes this nucleoprotein particle an attractive target for intervention. Apart from nucleic acid-based strategies $(19,20)$, dominant negative core protein variants have been described that passively interfere with nucleocapsid assembly (21-24). A conceptually more powerful approach is capsid-targeted viral inactivation (CTVI), or more generally, virion-targeted viral inactivation, which exploits a viral capsid protein or another virionassociated protein as a carrier to target a degradative enzyme specifically into virus particles $(25,26)$. Alternatively, nucleic acid-based effectors such as ribozymes may be fused to viral packaging signals and used against viruses that, like retroviruses but unlike $\mathrm{HBV}$, encapsidate more than one genome or genome segment $(27,28)$. For the protein-based approach, the ribonuclease from human eosinophil-derived neurotoxin (hEDN), which is an important member of the ribonuclease A superfamily, is considered particularly useful. It was discovered based upon a specific neurotoxicity to Purkinje cells and it differs markedly from RNase A in this activity $(29,30)$. EDN is found in cytotoxic granules located in eosinophils, and it may play a role in the anti-parasite and anti-tumor activities of eosinophils. It was reported that rEDN was not toxic to a variety of tumor cells in cultures up to $12 \mu \mathrm{M}$, whereas EDNsFv was specifically toxic to target cells at $1 \mathrm{pM}$. Therefore, EDN can be used to create specific and cytotoxic chimeric proteins (16).

The effector molecule in our study, hEDN, is a ribonuclease. Therefore, the target molecule degraded by the targeted ribonuclease constructed by us is most probably HBV pgRNA, the only RNA stage in the replication of HBV. As stated above, this notion is also supported by our experimental results, since the ribonuclease activity of hEDN is necessary for the anti-HBV effect of the TR. Similarly, onconase, an amphibian ribonuclease, was reported to inhibit HIV replication intracellularly by degrading HIV RNA (31). The degradation of HBV pgRNA not only leads to less mature virions released from host cells (which means secreted viral particles have lower infectivity), but it also inhibits the amplification of HBV closed circular DNA (cccDNA) which is downstream of pgRNA in HBV replication (32). This inhibition of HBV cccDNA amplification is of pivotal significance for the treatment of chronic HBV infection since the amplification of cccDNA, the template for all $\mathrm{HBV}$ transcripts, plays a major role in the persistence of $\mathrm{HBV}$ in infected hepatocytes $(1,33)$.

In summary, we constructed a novel targeted ribonuclease that specifically inhibits HBV replication but has no cytotoxicity for host cells. Our results raise the possibility of using this TR as a therapeutic agent for human HBV infection. For this purpose, we generated a recombinant adenovirus vector carrying the TR to test its antiviral efficacy in an HBV murine model and achieved beneficial results (34).

\section{Acknowledgements}

This study was supported by grants from the National Natural Science Foundation of China [grant no. 30100057 (2001)]. 


\section{References}

1. Ganem D and Varmus HE: The molecular biology of the hepatitis B viruses. Annu Rev Biochem 56: 651-693, 1987.

2. Lau JY and Wright TL: Molecular virology and pathogenesis of hepatitis B. Lancet 342: 1335-1340, 1993.

3. Tang ZY: Hepatocellular carcinoma - cause, treatment and metastasis. World J Gastroenterol 7: 445-454, 2001.

4. Hoofnagle JH: Therapy of viral hepatitis. Digestion 59: 563-578, 1998.

5. Hilleman MR: Overview of the pathogenesis, prophylaxis and therapeusis of viral hepatitis $\mathrm{B}$, with focus on reduction to practical applications. Vaccine 19: 1837-1848, 2001.

6. Francois G, Kew M, Van Damme P, Mphahlele MJ and Meheus A: Mutant hepatitis B viruses: a matter of academic interest only or a problem with far-reaching implications? Vaccine 19: 3799-3815, 2001.

7. Wong DK, Cheung AM, O'Rourke K, Naylor CD, Detsky AS and Heathcote $\mathrm{J}$ : Effect of alpha-interferon treatment in patients with hepatitis B e antigen-positive chronic hepatitis B. A metaanalysis. Ann Intern Med 119: 312-323, 1993.

8. Hoofnagle $\mathrm{JH}$ and di Bisceglie AM: The treatment of chronic viral hepatitis. N Engl J Med 336: 347-356, 1997.

9. Nevens F, Main J, Honkoop P, et al: Lamivudine therapy for chronic hepatitis B: a six-month randomized dose-ranging study. Gastroenterology 113: 1258-1263, 1997.

10. Tipples GA, Ma MM, Fischer KP, Bain VG, Kneteman NM and Tyrrell DL: Mutation in HBV RNA-dependent DNA polymerase confers resistance to lamivudine in vivo. Hepatology 24: 714-717, 1996.

11. Santantonio T, Mazzola M, Iacovazzi T, Miglietta A, Guastadisegni A and Pastore G: Long-term follow-up of patients with anti-HBe/HBV DNA-positive chronic hepatitis B treated for 12 months with lamivudine. J Hepatol 32: 300-306, 2000.

12. Tung FY and Bowen SW: Targeted inhibition of hepatitis B virus gene expression: a gene therapy approach. Front Biosci 3: a11-a15, 1998

13. Zhoug S, Wen SM, Zhang DF, Wang QL, Wang SQ and Ren H: Sequencing of PCR amplified HBV DNA pre-c and $\mathrm{c}$ regions in the 2.2 .15 cells and antiviral action by targeted antisense oligonucleotide directed against sequence. World J Gastroenterol 4: 434-436, 1998.

14. Liu J, Li YH, Xue CF, Ding J, Gong WD, Zhao Y and Huang YX: Targeted ribonuclease can inhibit replication of hepatitis $B$ virus. World J Gastroenterol 9: 295-299, 2003.

15. Buchner J, Pastan I and Brinkmann U: A method for increasing the yield of properly folded recombinant fusion proteins: singlechain immunotoxins from renaturation of bacterial inclusion bodies. Anal Biochem 205: 263-270, 1992.

16. Newton DL, Nicholls PJ, Rybak SM and Youle RJ: Expression and characterization of recombinant human eosinophil-derived neurotoxin and eosinophil-derived neurotoxin-anti-transferrin receptor sFv. J Biol Chem 269: 26739-26745, 1994.

17. Laras A, Koskinas J and Hadziyannis SJ: In vivo suppression of precore mRNA synthesis is associated with mutations in the hepatitis B virus core promoter. Virology 295: 86-96, 2002.

18. Wands JR, Geissler M, Putlitz JZ, et al: Nucleic acid-based antiviral and gene therapy of chronic hepatitis B infection. J Gastroenterol Hepatol 12: S354-S369, 1997.
19. Beck J and Nassal M: Hepatitis B virus replication. World J Gastroenterol 13: 48-64, 2007.

20. Scaglioni PP, Melegari M and Wands JR: Characterization of hepatitis B virus core mutants that inhibit viral replication. Virology 205: 112-120, 1994.

21. Scaglioni P, Melegari M, Takahashi M, Chowdhury JR and Wands J: Use of dominant negative mutants of the hepadnaviral core protein as antiviral agents. Hepatology 24: 1010-1017, 1996.

22. Von Weizsacker F, Wieland S and Blum HE: Inhibition of viral replication by genetically engineered mutants of the duck hepatitis B virus core protein. Hepatology 24: 294-299, 1996.

23. Von Weizsacker F, Kock J, Wieland S, Offensperger WB and Blum HE: Dominant negative mutants of the duck hepatitis B virus core protein interfere with RNA pregenome packaging and viral DNA synthesis. Hepatology 30: 308-315, 1999.

24. Natsoulis G and Boeke JD: New antiviral strategy using capsidnuclease fusion proteins. Nature 352: 632-635, 1991.

25. Boeke JD and Hahn B: Destroying retroviruses from within. Trends Microbiol 4: 421-426, 1996.

26. Giordano V, Jin DY, Rekosh D and Jeang KT: Intravirion targeting of a functional anti-human immunodeficiency virus ribozyme directed to pol. Virology 267: 174-184, 2000.

27. Sullenger BA and Cech TR: Tethering ribozymes to a retroviral packaging signal for destruction of viral RNA. Science 262: 1566-1569, 1993.

28. Tepper RI, Pattengale PK and Leder P: Murine interleukin-4 displays potent anti-tumor activity in vivo. Cell 57: 503-512, 1989.

29. Tepper RI, Coffman RL and Leder P: An eosinophil-dependent mechanism for the antitumor effect of interleukin-4. Science 257: 548-551, 1992.

30. Liu J, Li YH, Ding J, Gong WD, Xue CF, Zhao Y and Huang YX: Quantifying anti-HBV effect of targeted ribonuclease by real-time fluorescent PCR. World J Gastroenterol 10: 2883-2885, 2004.

31. Saxena SK, Gravell M, Wu YN, Mikulski SM, Shogen K, Ardelt W and Youle RJ: Inhibition of HIV-1 production and selective degradation of viral RNA by an amphibian ribonuclease. J Biol Chem 271: 20783-20788, 1996.

32. Tuttleman JS, Pourcel C and Summers J: Formation of the pool of covalently closed circular viral DNA in hepadnavirusinfected cells. Cell 47: 451-460, 1986.

33. Seeger C and Mason WS: Hepatitis B virus biology. Microbiol Mol Biol Rev 64: 51-68, 2000.

34. Zhao Y, Li Y, Liu J, et al: Adenoviral-vector mediated transfer of HBV-targeted ribonuclease can inhibit HBV replication in vivo. Biochem Biophys Res Commun 371: 541-545, 2008.

35. Rosenberg HF and Dyer KD: Eosinophil cationic protein and eosinophil-derived neurotoxin. Evolution of novel function in a primate ribonuclease gene family. J Biol Chem 270: 21539-21544, 1995.

36. Brinkmann U, Buchner $\mathrm{J}$ and Pastan I: Independent domain folding of Pseudomonas exotoxin and single-chain immunotoxins: influence of interdomain connections. Proc Natl Acad Sci USA 89: 3075-3079, 1992. 